\title{
Effect of high-fat diet feeding on hypothalamic redox signaling and central blood pressure regulation
}

\author{
Benedek Erdos $^{1,2}$, Christopher S Broxson ${ }^{1}$, Idan Cudykier ${ }^{1,2}$, Bilgen Basgut ${ }^{1,2}$, Melissa Whidden ${ }^{1,2}$, \\ Tessa Landa ${ }^{1,2}$, Philip J Scarpace ${ }^{2}$ and Nihal Tümer ${ }^{1,2}$
}

We examined the effect of high-fat (HF) feeding on blood pressure (BP) regulation, including hypothalamic redox signaling, as well as the changes in diurnal patterns and responses to restraint stress. Furthermore, we investigated whether HF feeding affects catecholamine and neuropeptide Y (NPY) biosynthesis in the adrenal medulla. Male obesity-prone Sprague-Dawley rats were fed with standard rat chow or $60 \% \mathrm{HF}$ diet for 6 months. BP and heart rate (HR) were measured by telemetry, and circadian changes as well as responses to $\mathbf{2 0}$ min restraint stress were analyzed. Mean arterial BP was significantly elevated in HF rats both during daytime and nighttime compared with controls, whereas HR was elevated only during the day. BP and HR increased similarly in response to stress in both experimental groups; however, post-stress recovery of BP and HR were significantly delayed in HF animals. Protein levels of angiotensin II type 1 receptor $\left(A T_{1}\right)$ and NOX2, p67 ${ }^{\text {phox }}$ and p47 $7^{\text {phox }}$ subunits of NADPH oxidase, as well as NADPH oxidase activity increased significantly in the hypothalamus with HF feeding, whereas levels of antioxidant enzymes and nitric oxide synthases remained unchanged. In addition, HF diet also elevated the adrenomedullary protein levels of tyrosine hydroxylase and NPY. This study shows that feeding obesity-prone Sprague-Dawley rats with a HF diet results in elevated BP and HR and delayed cardiovascular post-stress recovery, and that these changes are paralleled by increases in the expression and activity of NADPH oxidase in the hypothalamus without a compensatory increase in the antioxidant enzyme levels, possibly leading to superoxide-mediated sympathoexcitation and hypertension. Hypertension Research (2009) 32, 983-988; doi:10.1038/hr.2009.129; published online 28 August 2009

Keywords: angiotensin II; obesity; oxidative stress; restraint stress

\section{INTRODUCTION}

Obesity is the most prevalent nutritional disorder in industrialized countries, and it is associated with increased risk of hypertension and cardiovascular diseases. In addition, there is considerable evidence that activation of the sympathetic nervous system (SNS) has a key role in the pathogenesis of obesity-related hypertension both in experimental animals $^{1}$ and in humans. ${ }^{2}$

The brain renin-angiotensin system is an important regulator of SNS activity and arterial blood pressure. Central angiotensin II (AngII) enhances sympathetic outflow, blunts baroreflex sensitivity and stimulates secretion of vasopressin through its actions at various hypothalamic and medullary areas. ${ }^{3}$ In addition, AngII-mediated mechanisms seem to be involved in obesity-related hypertension. For instance, AngII type $1\left(\mathrm{AT}_{1}\right)$ receptor blockade normalizes the blood pressure of obesity-prone high-fat (HF) diet-fed animals, ${ }^{4}$ and increases in blood pressure in response to central AngII infusion are also significantly elevated in Zucker obese rats compared with lean animals. ${ }^{5}$

Superoxide anion has been indicated to mediate central AngIIinduced cardiovascular responses. For example, increases in blood pressure, heart rate and drinking elicited by the central injection of AngII were abolished by overexpression of superoxide dismutase (SOD) in the brain stem, ${ }^{6}$ and NADPH oxidase-dependent superoxide production has been shown to mediate AngII-induced responses both in the brain stem $^{7}$ and in the hypothalamus. ${ }^{8}$ Furthermore, sympathoexcitation in response to emotional stress ${ }^{9}$ and heart failure ${ }^{10}$ is also mediated by AngII and superoxide. Besides superoxide, nitric oxide (NO) has also been implicated in the regulation of SNS activity and blood pressure. Studies using NO synthase inhibitors suggest that tonic synthesis of NO inhibits SNS activity both in the brain stem and in the hypothalamus. ${ }^{11}$ On the other hand, overexpression of inducible NO synthase (iNOS) in the rostral ventrolateral medulla increases oxidative stress and elevates SNS activity. ${ }^{12}$ As obesity affects both redox signaling and NO synthesis throughout the body, ${ }^{13-15}$ there is a possibility that oxidative stress and reduced bioavailability of NO in cardiovascular regulatory nuclei mediate, at least in part, sympathoexcitation and blood pressure elevation in response to obesity.

Therefore, in this study, we examined the effect of HF feeding on central blood pressure regulation, including changes in diurnal

${ }^{1}$ Department of Veterans Affairs Medical Center, Geriatric Research, Education and Clinical Center, Gainesville, FL, USA and ${ }^{2}$ Department of Pharmacology and Therapeutics, University of Florida College of Medicine, Gainesville, FL, USA

Correspondence: Dr B Erdos, Department of Pharmacology \& Therapeutics, University of Florida, College of Medicine, PO Box 100267, Gainesville, FL 32610-0267, USA E-mail: erdos@ufl.edu

Received 2 December 2008; revised 15 July 2009; accepted 21 July 2009; published online 28 August 2009 
patterns and responses to restraint stress. In addition, we also tested whether HF diet-induced obesity affects hypothalamic AngII- and redox signaling, as well as expressions of neuronal and inducible NO synthases (nNOS and iNOS). Effects of HF diet were also evaluated on adrenomedullary expressions of tyrosine hydroxylase (TH) and neuropeptide Y (NPY), which have been shown to correlate with SNS activity. TH is the rate-limiting enzyme in catecholamine biosynthesis, whereas NPY is coreleased with catecholamines and has an important role in the regulation of catecholamine biosynthesis. ${ }^{16}$

\section{METHODS}

\section{Experimental animals}

Experiments were conducted according to the Guiding Principles in the Care and Use of Laboratory Animals, and procedures were approved by the local Institutional Animal Care and Use Committee. Male obesity-prone SpragueDawley rats were purchased from Charles River (Boston, MA, USA). This animal strain was developed by selective breeding of outbred Sprague-Dawley rats for elevated body weight gain on a high-energy diet. These animals develop obesity and metabolic syndrome with insulin resistance, impaired glucose tolerance and dyslipidemia when fed with a HF diet. ${ }^{17}$ Animals were acquired at 6 weeks of age, and were fed ad libitum either with standard rat chow (15\% fat; $3.3 \mathrm{kcal} \mathrm{g}^{-1}$; diet-2018; Harlan Teklad, Madison, WI) or a HF diet (60\% fat; $5.2 \mathrm{kcal} \mathrm{g}^{-1}$, D12492; Research Diets, New Brunswick, NJ, USA). After 3 months of diet, radiotelemetric probes were implanted with the cannulation of the femoral artery for the measurements of blood pressure and heart rate. Rats were housed individually with a 12:12 h light/dark cycle (0700 to $1900 \mathrm{~h}$ ).

\section{Radiotelemetry}

The right femoral artery was cannulated with the catheter of the transmitter (model PA-C40; Data Sciences International, St Paul, MN, USA) under isoflurane anesthesia, and the transmitter was placed in a subcutaneous pocket. Blood pressure and HR measurements started after 4 weeks of recovery from surgery using the telemetry system (Data Sciences International), and these parameters were measured for 2 months. Locomotor activity was also calculated using the telemetry system from a variation in signal strength.

\section{Restraint stress}

Two months after surgery (after 5 months of HF feeding), MABP and HR changes in response to stress were evaluated by placing the animals in acrylic cylinder restrainers for $20 \mathrm{~min}$. MABP and HR were measured for $30 \mathrm{~min}$ before stress, during the 20-min stress period and for $30 \mathrm{~min}$ after stress.

\section{Tissue harvesting and preparation}

Animals were over-anesthetized with pentobarbital $\left(120 \mathrm{mg} \mathrm{kg}^{-1}\right.$ i.p.); the adrenal glands and the hypothalamus were removed quickly and frozen immediately in liquid nitrogen. At the time of the assay, the adrenal glands were decapsulated and the medullae were separated from the cortex. The adrenal medullae and the hypothalamus were homogenized in phosphate buffer (2 mm NaPO4, 0.2\% Triton, pH 7.0).

\section{Northern blot}

The TH and NPY mRNA levels were determined using northern blot as previously described. ${ }^{18}$ Briefly, total cellular RNA was extracted using Trireagent (Sigma, St Louis, MO, USA), and the isolated RNA was quantified by spectrophotometry. Membranes were hybridized with ${ }^{32} \mathrm{P}$ random primergenerated probes. After hybridization, the membranes were washed and exposed to the phospho screen for $24 \mathrm{~h}$ using a Phospho Imager (Molecular Dynamics, Sunnyville, CA, USA). Screens were scanned and analyzed using Image Quant software (Molecular Dynamics).

\section{Western blot}

$\mathrm{TH}, \mathrm{AT}_{1}$ receptor, NOX2 (NADPH oxidase subunit-2), $\mathrm{p}^{\text {phox }}$, p67 $7^{\text {phox }}$ protein levels were determined using western blot as previously described. ${ }^{18}$ Briefly, an equal amount of protein for each sample was separated by $12.5 \%$ SDS-PAGE, transferred onto a nitrocellulose membrane and blocked with 5\% skimmed milk in Tris-buffered saline containing $0.1 \%$ Tween 20 . Blots were incubated overnight at $4{ }^{\circ} \mathrm{C}$ with anti-TH (Pel-Freez Biologicals, Rogers, AR, USA), anti$\mathrm{AT}_{1}$ (Fitzgerald Industries, Concord, MA, USA), anti-gp91/NOX2 (BD Transduction Labs, San Diego, CA, USA), and anti-p47 $7^{\text {phox }}$ and anti-p67 $7^{\text {phox }}$ (Upstate, Temecula, CA, USA) antibodies. Bound antibodies were detected by chemifluorescence.

\section{NADPH oxidase activity}

NADPH oxidase activity was measured with a lucigenin-enhanced chemiluminescence assay using hypothalamus homogenates. Tissue samples were incubated in artificial CSF at $37^{\circ} \mathrm{C}$ in a luminometer (BMG Fluostar Optima, Offenburg, Germany). Scintillation counts were obtained for $30 \mathrm{~min}$ in the presence of NADH $(100 \mu \mathrm{M})$ and lucigenin $(5 \mu \mathrm{M})$, and background-corrected values were normalized to protein content.

\section{Characterization of the metabolic effects of HF feeding}

Blood samples were taken by cardiac puncture from non-fasted animals at the time of killing. Plasma insulin levels were measured using a rat insulin enzymelinked immunosorbent assay (ELISA) kit (Millipore, Billerica, MA, USA), and glucose levels were assessed using a kit from Biovision (Mountain View, CA, USA), whereas total cholesterol and triglyceride levels were measured by assays from Wako Diagnostics (Richmond, VA, USA).

\section{Statistical analysis}

Data were expressed as means \pm s.e. Differences between experimental groups were evaluated using analysis of variance, followed by Tukey's post hoc test. The criterion for significance was $P<0.05$.

\section{RESULTS}

Body weight of HF diet-fed rats increased more rapidly compared with controls, and became significantly elevated after 10 weeks of diet (Figure 1). Rats in both groups lost weight after telemetric surgery; however, following 4 weeks of recovery, rats regained their pre-surgery body weights. By the 18th week of diet feeding, HF rats again weighed significantly more than control rats, and at the end of the 24 -week diet treatment, HF-fed animals weighed $728 \pm 11 \mathrm{~g}(n=6)$ compared with chow-fed animals, $634 \pm 15 \mathrm{~g}(n=6, P<0.01$; Figure 1$)$. In addition, HF feeding also led to dyslipidemia. Both non-fasting total cholesterol and triglycerides were significantly elevated in HF rats (Table 1). In contrast, non-fasting insulin and glucose levels were similar in the control and HF groups.

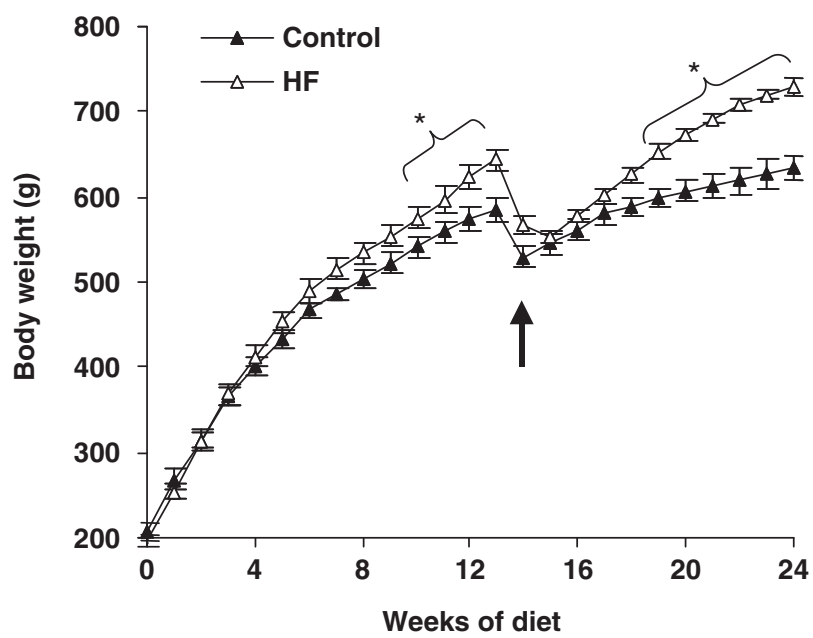

Figure 1 Effect of diet feeding on body weight. HF feeding was initiated at 6 weeks of age; arrow indicates weight drop following radiotelemetric transmitter implantation. Values represent mean \pm s.e., ${ }^{*} P<0.05$. 
Table 1 Metabolic effects of HF diet feeding

\begin{tabular}{lcccc}
\hline & Glucose $(\mathrm{mg}$ per $100 \mathrm{ml})$ & Insulin $(\mathrm{ng} / \mathrm{ml})$ & Total cholesterol $(\mathrm{mg}$ per 100 ml) & Triglycerides $(\mathrm{mg}$ per 100 ml) \\
\hline Control & $140.1 \pm 3.1$ & $1.91 \pm 0.49$ & $106.2 \pm 4.67$ & $102.0 \pm 10.7$ \\
High fat & $139.8 \pm 4.0$ & $2.19 \pm 0.46$ & $155.3 \pm 22.18^{\mathrm{a}}$ & $197.3 \pm 8.0^{\mathrm{a}}$ \\
\hline
\end{tabular}

a $P<0.05$ High fat vs. control.
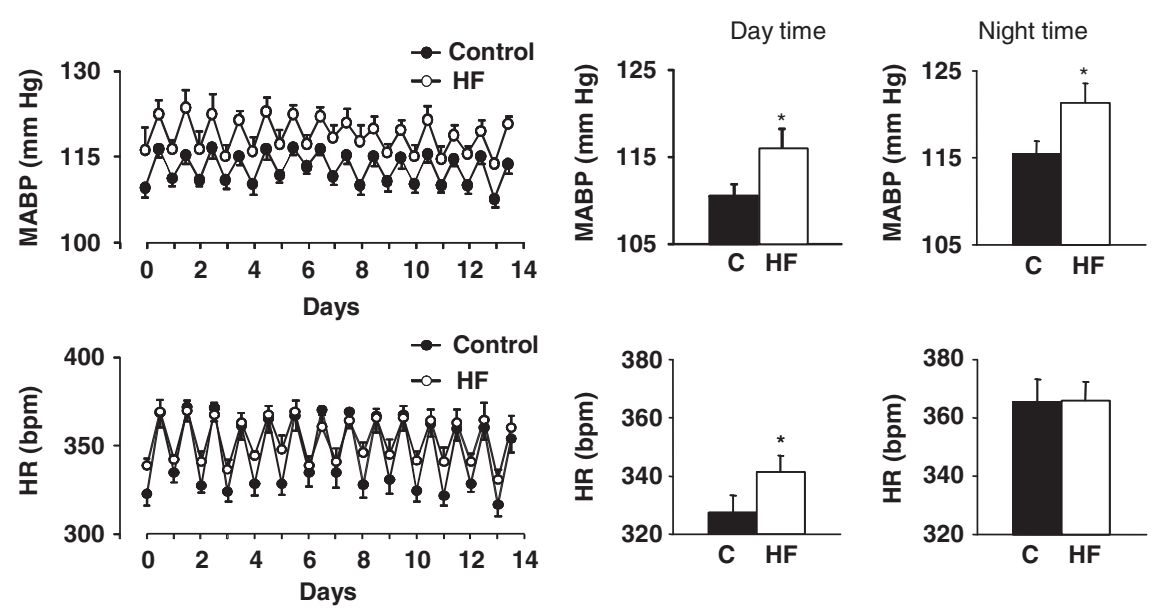

Figure 2 Average mean arterial blood pressure (MABP) and heart rate (HR) values from lean and HF diet-fed animals. Left panel: continuous recordings from the last 2 weeks of the experiment. Right panel, group averages calculated from measurements during the last 2 months of the experiment. HF diet feeding significantly elevated MABP both during the day and during the night, whereas HR was elevated only during the day. Values represent mean \pm s.e., ${ }^{*} P<0.05$.

Resting blood pressure, heart rate and locomotor activity Blood pressure values were significantly elevated in the HF group compared with controls. In control rats, daytime mean arterial blood pressure (MABP), diastolic blood pressure (DBP) and systolic blood pressure (SBP) were $110.6 \pm 1.4,95.6 \pm 1.8$ and $133.1 \pm 2.0 \mathrm{~mm} \mathrm{Hg}$, respectively, whereas in HF animals, daytime MABP, DBP and SBP were $116.1 \pm 2.2,101.3 \pm 3.4$ and $138.3 \pm 2.6 \mathrm{~mm} \mathrm{Hg}$ (Figure 2, $P<0.05$ vs. control for all three parameters). Nighttime blood pressure levels were similarly elevated in HF animals (MABP, DBP and SBP were $121.3 \pm 2.3,105.5 \pm 3.3$ and $144.2 \pm 2.7 \mathrm{~mm} \mathrm{Hg}$ ) compared with controls, where MABP, DBP and SBP were $115.5 \pm 1.4,100.1 \pm 1.6$ and $138.4 \pm 2.1 \mathrm{~mm} \mathrm{Hg}$, respectively (Figure $2, P<0.05$ for all three parameters). In contrast, HR was elevated in the HF animals only during the day ( $341 \pm 6$ b.p.m. compared with $327 \pm 6$ b.p.m. in controls, $P<0.05)$, whereas nighttime HR levels were similar in the control (365 \pm 8 b.p.m.) and HF groups ( $367 \pm 7$ b.p.m., Figure 2). These differences in MABP and HR were already present at the start of telemetric measurements (after 4 months of HF diet), and there was no significant change during the 2-month measurement period. Locomotor activity levels, measured as changes in signal strength by the telemetry system, were similar between control and HF rats during the day ( $48 \pm 11 \mathrm{AU} v s .49 \pm 11 \mathrm{AU}, P=0.90)$, and although activity levels were slightly reduced in the HF group compared with controls during the night, this difference was not statistically significant $(160 \pm 15 \mathrm{AU}$ vs. $186 \pm 21 \mathrm{AU}, P=0.51)$.

\section{Cardiovascular stress responses}

MABP and HR increased rapidly in response to stress in both experimental groups, with similar peak values of MABP and HR in the control and HF rats (Figure 3). MABP began to decline during stress both in the control and HF animals, but the post-stress recovery of MABP was significantly slower in the HF animals. Although MABP returned to pre-stress values in the control rats in $15 \mathrm{~min}$, it remained elevated till the end of post-stress measurements in the HF group. HR also started to decline during stress in the control group, but remained elevated in the HF rats, resulting in significantly higher HR during the second part of stress. Interestingly, there was a second peak in the HR recordings at the end of the stress period, elicited most likely by the stress associated with the removal of animals from the restrainers. During the post-stress period, HR returned to normal levels in about $15 \mathrm{~min}$ in the control group, whereas remaining significantly elevated in the HF group till the end of post-stress measurements (Figure 3).

\section{Hypothalamic effects of HF feeding}

$\mathrm{AT}_{1}$ receptor expression increased significantly in the hypothalamus in response to $\mathrm{HF}$ diet feeding, and the $\mathrm{AT}_{1}$ receptor protein level was $19 \pm 5 \%$ higher in the HF group compared with controls (Figure 4, $P<0.05)$. In addition, the hypothalamic levels of NADPH oxidase subunits were also elevated in the HF-fed animals. The NOX2 protein level was more than twofold higher in the HF group compared with controls $(P<0.01)$, whereas regulatory subunits, p6 $7^{\text {phox }}$ and $\mathrm{p} 47^{\mathrm{phox}}$, increased by $19 \pm 6 \%(P<0.05)$ and $30 \pm 6 \%$, respectively (Figure 4 , $P<0.05)$. In concert with these changes, NADPH oxidase activity was also elevated by HF feeding in the hypothalamus, and was $78 \pm 23 \%$ higher in HF rats compared with controls (Figure 5, $P<0.05$ ). In contrast, HF feeding did not significantly affect the levels of antioxidant enzymes. CuZnSOD protein level decreased by $13 \pm 10 \%$ $(P=0.36)$, MnSOD protein level decreased by $14 \pm 9 \%(P=0.30)$, whereas catalase remained unchanged $(-1 \pm 9 \%, P=0.91)$ in $\mathrm{HF}$ rats compared with controls. Protein levels of nNOS and iNOS were also evaluated in the hypothalamus, however, HF diet feeding did not alter the expression of these enzymes either. nNOS and iNOS changed 

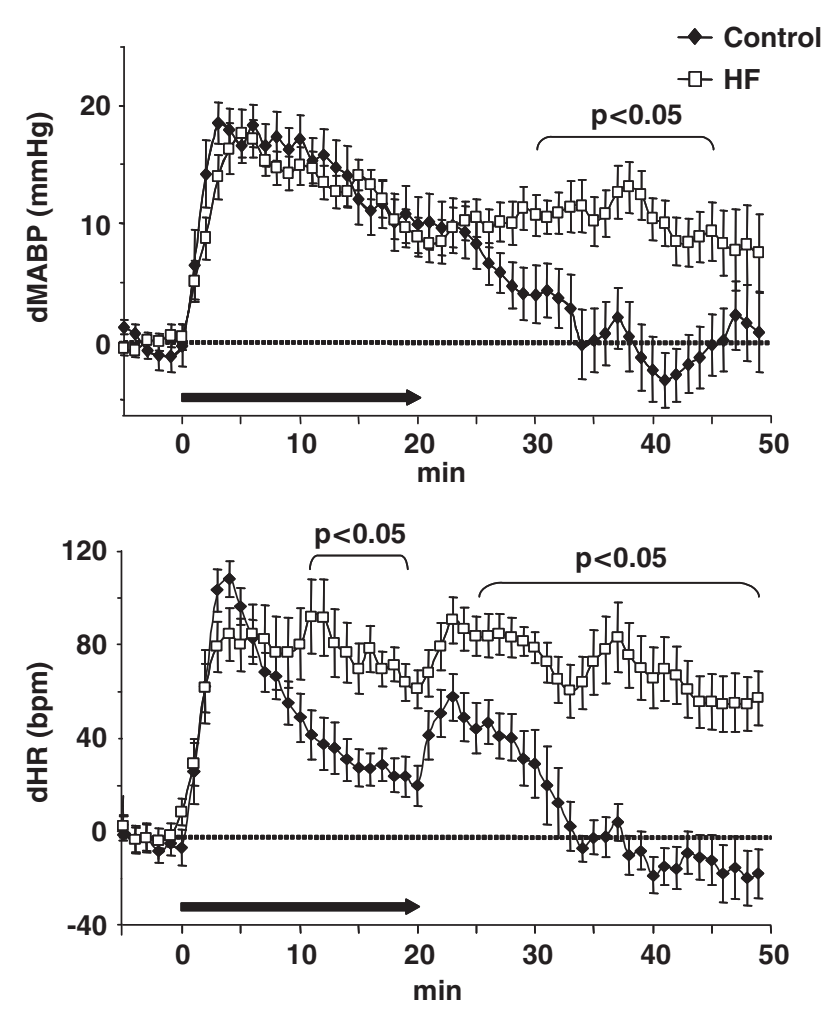

Figure 3 Changes in mean arterial blood pressure (dMABP) and heart rate (dHR) before, during and after the 20 min restraint stress. MABP and HR increased similarly in both the control and HF groups; however, recovery from stress was delayed in the HF diet-fed animals. Values represent mean \pm s.e., and arrow represents the duration of stress.
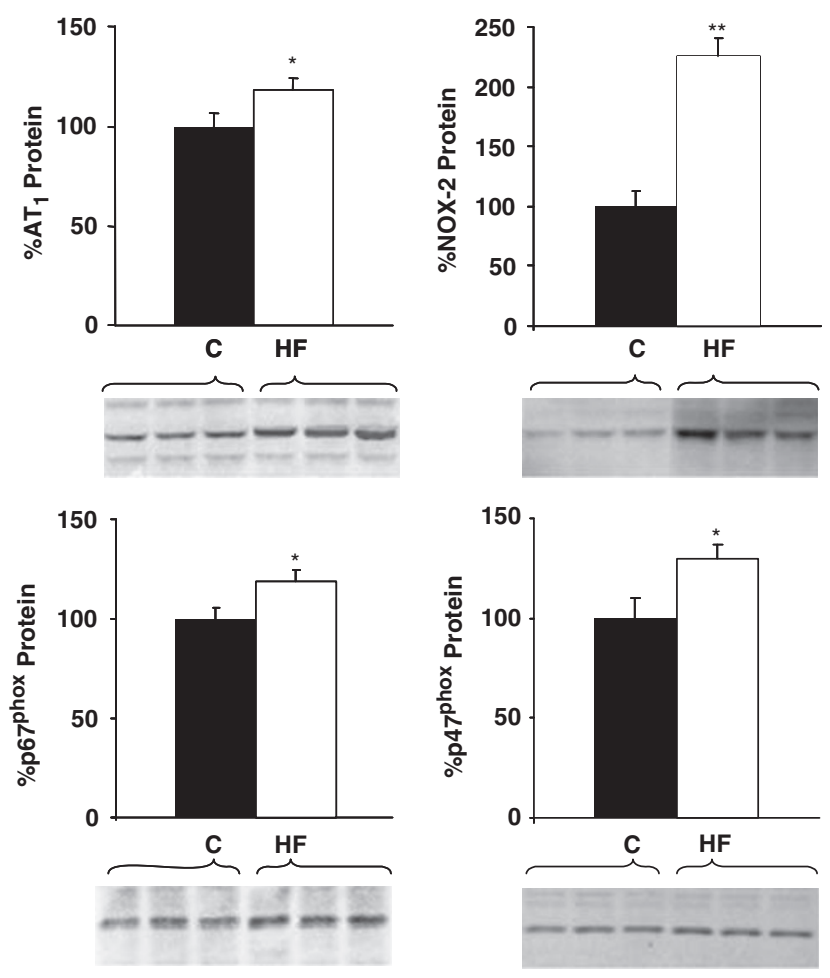

Figure 4 Effects of $\mathrm{HF}$ feeding on hypothalamic protein levels of $\mathrm{AT}_{1}$ receptor and NADPH oxidase subunits as measured with western blot analysis and expressed as percentage of control. Values represent mean \pm s.e., ${ }^{*} P<0.05 ;{ }^{*} P<0.01$.

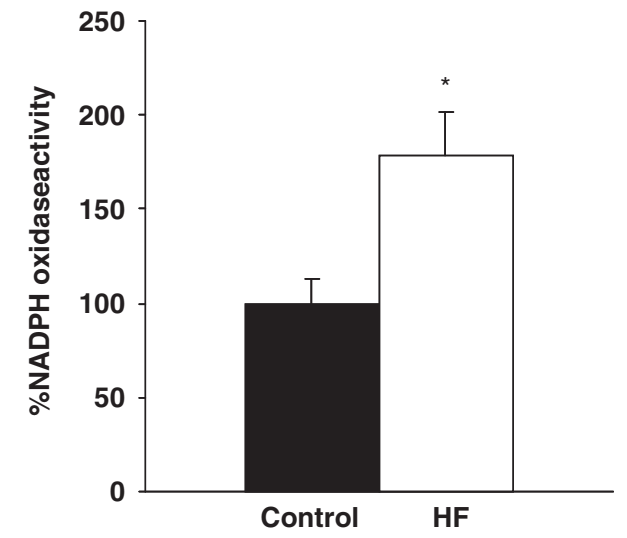

Figure 5 Effects of HF feeding on hypothalamic NADPH oxidase activity as measured with lucigenin-enhanced chemiluminescence. Scintillation counts were normalized by protein content of hypothalamic samples, and expressed as percentage of control. Values represent mean \pm s.e., ${ }^{*} P<0.05$.
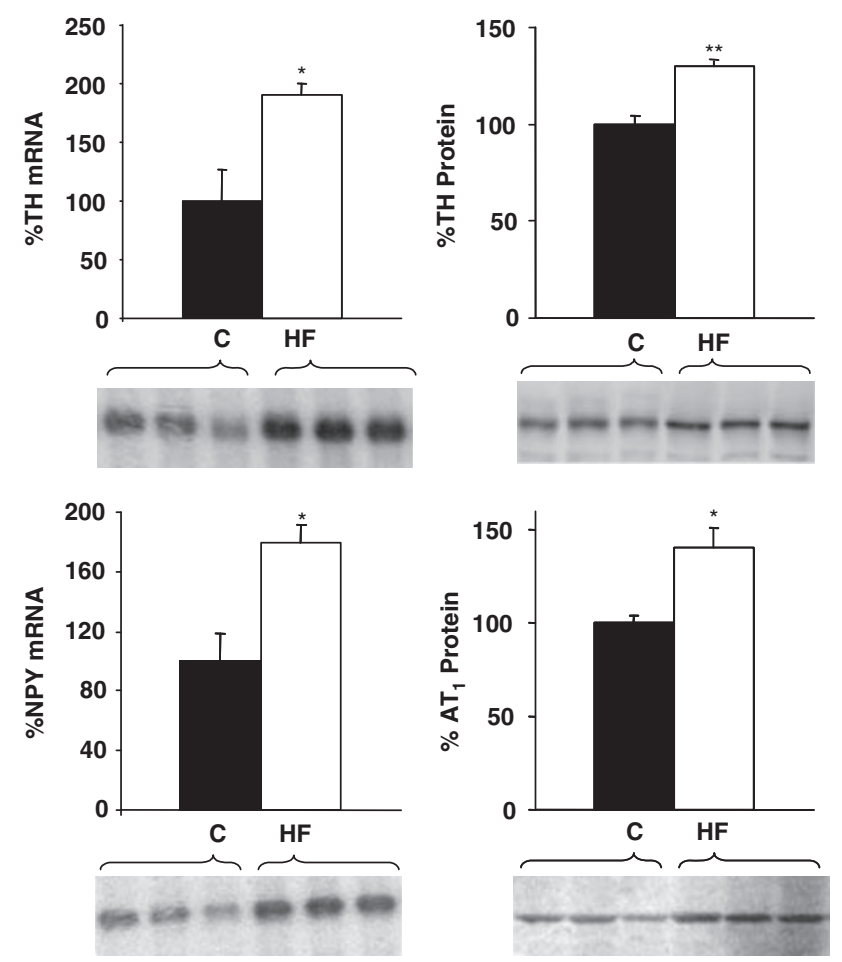

Figure 6 Effects of HF feeding on adrenomedullary expression of TH, NPY and $\mathrm{AT}_{1}$ receptors as measured with western blot and northern blot, and expressed as percentage of control. Values represent mean \pm s.e., ${ }^{*} P<0.05$; $* * P<0.01$.

by $5 \pm 3 \% \quad(P=0.20)$ and $-2 \pm 6 \% \quad(P=0.81)$ in the HF group compared with controls.

\section{HF diet-induced changes in the adrenal medulla}

TH expression increased significantly in the adrenal medulla in response to HF diet feeding, and the TH mRNA and protein levels were elevated by $90 \pm 11 \%(P<0.05)$. and $29 \pm 3 \%(P<0.05)$ in the HF group compared with controls. In addition, NPY mRNA increased by $80 \pm 11 \%(P<0.05)$, whereas $\mathrm{AT}_{1}$ receptor protein levels increased by $43 \pm 13 \%$ in the adrenal medulla of HF animals compared with controls (Figure 6, $P<0.05$ ). 


\section{DISCUSSION}

The major results of this study are that HF diet feeding increases the hypothalamic expression of $\mathrm{AT}_{1}$ receptors and subunits of NADPH oxidase in obesity-prone Sprague-Dawley rats, whereas the levels of antioxidant enzymes as well as nNOS and iNOS remain unaltered. HF feeding also increases the protein levels of $\mathrm{AT}_{1}$ receptors in the adrenal medulla, and elevates the adrenomedullary TH and NPY expressions. These hypothalamic and adrenomedullary changes are paralleled by significant increases in day- and nighttime blood pressure and daytime $\mathrm{HR}$ in HF animals, as well as delayed recovery from restraint stressinduced blood pressure and HR elevations.

Obesity is a well-known risk factor for hypertension in humans, and HF diet-induced obesity increases the blood pressure in several animal models including dogs ${ }^{19}$ and rabbits. ${ }^{20}$ However, HF feeding in rats led to mixed results. Although some studies reported significant increases in blood pressure using tail cuff measurements, ${ }^{21,22}$ others that used telemetry showed no change. ${ }^{23}$ In addition, the question was raised that higher blood pressure values measured with tail cuff in HFfed animals were the result of an augmented stress response to restraint accompanying this blood pressure measurement procedure. This explanation is supported by those findings where obesity leads to amplified and longer lasting cardiovascular stress responses in obese Zucker rats ${ }^{24}$ and in spontaneously hypertensive rats fed with an HF diet. ${ }^{25}$ Therefore, in this study, we decided to test whether blood pressure, measured in freely moving obesity-prone rats, can be increased by a diet with higher fat content $(60 \%$ of total calorie), fed for a longer period ( 24 weeks) compared with the $32 \%$ HF diet fed for $10-12$ weeks in earlier rat studies. ${ }^{21-23}$ This diet treatment led to significantly elevated body weight and dyslipidemia; however, the plasma insulin and glucose levels were not elevated compared with chow-fed rats. This could be because both our control and HF diet-fed rats were selectively bred obesity-prone rats that show signs of metabolic syndrome even when fed with regular chow. ${ }^{17}$ Furthermore, our results indicate that this more robust diet treatment leads to cardiovascular changes that resemble those observed in humans and other experimental models. Blood pressure increased significantly, and the diurnal pattern of $\mathrm{HR}$ was also disrupted; therefore, the reduction of HR during the resting daytime period became significantly attenuated.

Reduced dipping of blood pressure and HR has been observed in hypertensive humans, and it has been associated with a higher incidence of left ventricular hypertrophy, microalbuminuria, cerebrovascular diseases, and stroke. ${ }^{26-28}$ In addition, reduced dipping was also observed in type II diabetic patients, ${ }^{29}$ as well as in rabbits fed with an HF diet. ${ }^{20}$ The circadian variation of blood pressure is thought to be controlled by the autonomic nervous system, and a non-dipping pattern is usually correlated with higher SNS activity or reduced parasympathetic activity and hypertension. ${ }^{30}$ Interestingly, in our model, only the circadian rhythm of HR was affected, whereas the pattern of blood pressure was maintained despite higher diurnal and nocturnal values. Antic et al. ${ }^{20}$ have shown in HF diet-fed rabbits that circadian changes in HR and blood pressure are regulated differently; however, further studies are needed to examine why the patterns of blood pressure and HR are influenced differently in the rat. Nevertheless, our results suggest that nighttime BP remains significantly elevated in HF rats despite normal nighttime HR values because of vasoconstriction and an increase in peripheral vascular resistance.

In addition to elevated blood pressure and changes in the circadian pattern of HR, our study has also shown that post-stress cardiovascular recovery is delayed in HF diet-fed animals. These data confirm earlier findings from studies in Zucker obese and spontaneously hypertensive rats. ${ }^{24,25}$ Such delays in cardiovascular recovery from stress could have an important role in the development of obesity hypertension, as adaptation to stressful stimuli from the environment is part of everyday life, and dietary obesity may significantly potentiate the impact of daily stressful experiences on the cardiovascular system. The importance of this effect of diet-induced obesity is further supported by those studies that showed that delayed cardiovascular post-stress recovery is associated with elevated risk of hypertension. ${ }^{31}$ Although the underlying mechanisms are not completely understood, a proposed mechanism is that $\beta$-adrenergic vasodilation, which seems to have a key role in lowering blood pressure after stress in lean animals, is reduced in the obese. ${ }^{24}$ However, data from our study show that besides blood pressure, the recovery of HR is also delayed. This suggests that central SNS regulation is affected by HF feeding, too, and our results indicate that one possible way how diet-induced obesity may affect blood pressure regulation is through altered central redox signaling.

Zanzinger et al. ${ }^{32}$ have shown that oxidative stress in the brain stem increases SNS activity, heart rate and blood pressure, which can be reversed with local injections of SOD. In addition, reports indicate that redox signaling mediates central AngII-induced cardiovascular responses. ${ }^{6-10}$ As obesity is associated with systemic oxidative stress, ${ }^{13,33}$ we hypothesized that HF diet-induced obesity could increase superoxide production and/or reduce antioxidant capacity in the brain resulting in elevated SNS activity and blood pressure. In this study, we found that NOX2, the main membrane-associated subunit of NADPH oxidase, and two regulatory subunits, $\mathrm{p} 4 \mathrm{7ph}^{\mathrm{phox}}$ and $\mathrm{p} 67^{\mathrm{phox}}$, are significantly upregulated in response to HF feeding in the hypothalamus resulting in increased NADPH oxidase activity. In contrast, antioxidant enzymes such as MnSOD, CuZnSOD and catalase are unaffected. These results suggest that there might be an imbalance between production and elimination of reactive oxygen species in the hypothalamus of HF diet-fed animals, which may contribute to elevated SNS activity and BP. A possible mechanism underlying the upregulation of NADPH oxidase is increased Ang II signaling. ${ }^{10}$ Our results show that HF feeding significantly increases $\mathrm{AT}_{1}$ receptor expression in the hypothalamus, and this change in the expressions of $\mathrm{AT}_{1}$ receptor and NADPH oxidase could also explain why pressor responses to centrally infused AngII are elevated in obese animals. ${ }^{5}$ Moreover, as central AngII and NADPH oxidase-dependent superoxide production have a significant role in the mediation of stress-induced pressor responses, ${ }^{9}$ the upregulation of $\mathrm{AT}_{1}$ receptor and NADPH oxidase without a compensatory increase in antioxidant enzymes could mean that turning off this sympathoexcitatory pathway may take longer in obese animals resulting in the observed delay in post-stress recovery of blood pressure and HR. In this study, we only analyzed changes in hypothalamic redox signaling, but similar changes in the AngII-NADPH oxidase pathway may occur in other regulatory nuclei, such as the nucleus tractus solitarius or the rostral ventrolateral medulla. Further studies will be needed to clarify whether dietinduced obesity affects these different brain regions similarly causing autonomic dysregulation at multiple sites.

The rate of adrenomedullary catecholamine biosynthesis has been shown to correlate with SNS activity. For example, we and others have previously shown that $\mathrm{TH}$, the rate-limiting enzyme in catecholamine biosynthesis, is upregulated in the adrenal medulla in response to stress, ${ }^{34}$ central AngII infusion ${ }^{35}$ and also with age. ${ }^{36}$ Thus, in this study, we examined whether HF diet feeding is also associated with an increase in adrenomedullary TH protein levels. Our data show that both the mRNA and protein expressions of TH increased significantly in response to HF feeding. In addition, we have shown that NPY, 
which is coreleased with catecholamines in the adrenal medulla, was also upregulated in the HF diet-fed animals. Besides the increased SNS activity, peripheral mechanisms, such as the systemic renin-angiotensin system, may also influence adrenomedullary catecholamine biosynthesis. Both the $\mathrm{AT}_{1}$ and Ang type- 2 receptors are expressed in the adrenal medulla, but the activation of $\mathrm{AT}_{1}$ receptors seem to mediate the upregulation and activation of adrenomedullary $\mathrm{TH}^{37,38}$ In this study, we found that the $\mathrm{AT}_{1}$ receptor was upregulated in the HF diet-fed animals. This finding, together with those previous reports that the systemic renin-angiotensin system is activated by HF feeding, ${ }^{22}$ suggests that AngII may contribute to the upregulation of catecholamine biosynthetic enzymes in the adrenal medulla, and to the elevated plasma catecholamine levels observed in obese subjects. ${ }^{39}$

In conclusion, this study shows that feeding obesity-prone SpragueDawley rats with a $60 \%$ HF diet for 24 weeks results in elevated BP, reduced daytime HR dipping and delayed cardiovascular post-stress recovery. In addition, $\mathrm{HF}$ diet feeding upregulates $\mathrm{AT}_{1}$ receptors and NADPH oxidase subunits in the hypothalamus with a concomitant increase in NADPH oxidase-dependent superoxide production, possibly leading to superoxide-mediated sympathoexcitation and hypertension.

\section{CONFLICT OF INTEREST}

The authors declare no conflict of interest.

\section{ACKNOWLEDGEMENTS}

This study was supported by the Medical Research Service of the Department of Veterans Affairs and by an award from the American Heart Association. Bilgen Basgut was supported by a TUBITAK scholarship, and Melissa Whidden was supported by an NIA T32 training grant (AG000196).

1 Barnes MJ, Lapanowski K, Conley A, Rafols JA, Jen KL, Dunbar JC. High fat feeding is associated with increased blood pressure, sympathetic nerve activity and hypothalamic mu opioid receptors. Brain Res Bull 2003; 61: 511-519.

2 Vaz M, Jennings G, Turner A, Cox H, Lambert G, Esler M. Regional sympathetic nervous activity and oxygen consumption in obese normotensive human subjects. Circulation 1997; 96: 3423-3429.

3 Phillips MI, Sumners C. Angiotensin II in central nervous system physiology. Regul Pept 1998; 78: 1-11.

4 Boustany CM, Brown DR, Randall DC, Cassis LA. AT1-receptor antagonism reverses the blood pressure elevation associated with diet-induced obesity. Am J Physiol Regul Integr Comp Physiol 2005; 289: R181-R186.

5 Bokil HS, Porter JP. Brain angiotensin type 1 receptor expression and function in the Zucker obese rat. Neurosci Lett 2000; 281: 139-142.

6 Zimmerman MC, Lazartigues E, Lang JA, Sinnayah P, Ahmad IM, Spitz DR, Davisson RL. Superoxide mediates the actions of angiotensin II in the central nervous system. Circ Res 2002; 91: 1038-1045.

7 Wang G, Anrather J, Huang J, Speth RC, Pickel VM, ladecola C. NADPH oxidase contributes to angiotensin II signaling in the nucleus tractus solitarius. J Neurosci 2004; 24: 5516-5524.

8 Erdos B, Broxson CS, King MA, Scarpace PJ, Tumer N. Acute pressor effect of central angiotensin II is mediated by NAD(P)H-oxidase-dependent production of superoxide in the hypothalamic cardiovascular regulatory nuclei. J Hypertens 2006; 24: 109-116.

9 Mayorov DN, Head GA, De Matteo R. Tempol attenuates excitatory actions of angiotensin II in the rostral ventrolateral medulla during emotional stress. Hypertension 2004; 44: 101-106.

10 Gao L, Wang W, Li YL, Schultz HD, Liu D, Cornish KG, Zucker IH. Sympathoexcitation by central ANG II: roles for AT1 receptor upregulation and NAD(P)H oxidase in RVLM. Am J Physiol Heart Circ Physiol 2005; 288: H2271-H2279.

11 Zanzinger J. Mechanisms of action of nitric oxide in the brain stem: role of oxidative stress. Auton Neurosci 2002; 98: 24-27.

12 Kimura Y, Hirooka Y, Sagara Y, Ito K, Kishi T, Shimokawa H, Takeshita A, Sunagawa K. Overexpression of inducible nitric oxide synthase in rostral ventrolateral medulla causes hypertension and sympathoexcitation via an increase in oxidative stress. Circ Res 2005; 96: 252-260.
13 Dobrian AD, Davies MJ, Schriver SD, Lauterio TJ, Prewitt RL. Oxidative stress in a rat model of obesity-induced hypertension. Hypertension 2001; 37: 554-560.

14 Stepp DW. Impact of obesity and insulin resistance on vasomotor tone: nitric oxide and beyond. Clin Exp Pharmacol Physiol 2006; 33: 407-414.

15 Erdos B, Snipes JA, Miller AW, Busija DW. Cerebrovascular dysfunction in zucker obese rats is mediated by oxidative stress and protein kinase C. Diabetes 2004; 53 : $1352-1359$

16 Hong M, Li S, Fournier A, St-Pierre S, Pelletier G. Role of neuropeptide $Y$ in the regulation of tyrosine hydroxylase gene expression in rat adrenal glands. Neuroendocrinology 1995; 61: 85-88.

17 Levin BE, Dunn-Meynell AA, Balkan B, Keesey RE. Selective breeding for diet-induced obesity and resistance in Sprague-Dawley rats. Am J Physiol 1997; 273: R725-R730.

18 Erdos B, Broxson CS, Landa T, Scarpace PJ, Leeuwenburgh C, Zhang Y, Tumer N. Effects of life-long caloric restriction and voluntary exercise on age-related changes in levels of catecholamine biosynthetic enzymes and angiotensin II receptors in the rat adrenal medulla and hypothalamus. Exp Gerontol 2007; 42: 745-752.

19 Truett AA, Borne AT, Poincot MA, West DB. Autonomic control of blood pressure and heart rate in obese hypertensive dogs. Am J Physiol 1996; 270: R541-R549.

20 Antic V, Van Vliet BN, Montani JP. Loss of nocturnal dipping of blood pressure and heart rate in obesity-induced hypertension in rabbits. Auton Neurosci 2001; 90: 152-157.

21 Dobrian AD, Davies MJ, Prewitt RL, Lauterio TJ. Development of hypertension in a rat model of diet-induced obesity. Hypertension 2000; 35: 1009-1015.

22 Boustany CM, Bharadwaj K, Daugherty A, Brown DR, Randall DC, Cassis LA. Activation of the systemic and adipose renin-angiotensin system in rats with diet-induced obesity and hypertension. Am J Physiol Regul Integr Comp Physiol 2004; 287: R943-R949.

23 Carroll JF, Zenebe WJ, Strange TB. Cardiovascular function in a rat model of dietinduced obesity. Hypertension 2006; 48: 65-72.

24 D'Angelo G, Mintz JD, Tidwell JE, Schreihofer AM, Pollock DM, Stepp DW. Exaggerated cardiovascular stress responses and impaired beta-adrenergic-mediated pressor recovery in obese Zucker rats. Hypertension 2006; 48: 1109-1115.

25 Sedova L, Berube J, Gaudet D, Dumont M, Tremblay J, Hamet P, Pausova Z. Dietinduced obesity delays cardiovascular recovery from stress in spontaneously hypertensive rats. Obes Res 2004; 12: 1951-1958.

26 Bianchi S, Bigazzi R, Baldari G, Sgherri G, Campese VM. Diurnal variations of blood pressure and microalbuminuria in essential hypertension. Am J Hypertens 1994; 7: 23-29.

27 Verdecchia P, Schillaci G, Guerrieri M, Gatteschi C, Benemio G, Boldrini F, Porcellati C. Circadian blood pressure changes and left ventricular hypertrophy in essential hypertension. Circulation 1990; 81: 528-536.

28 Shimada K, Kawamoto A, Matsubayashi K, Nishinaga M, Kimura S, Ozawa T. Diurnal blood pressure variations and silent cerebrovascular damage in elderly patients with hypertension. J Hypertens 1992; 10: 875-878.

29 Nakano S, Fukuda M, Hotta F, Ito T, Ishii T, Kitazawa M, Nishizawa M, Kigoshi T, Uchida K. Reversed circadian blood pressure rhythm is associated with occurrences of both fatal and nonfatal vascular events in NIDDM subjects. Diabetes 1998; 47: 1501-1506.

30 Kohara K, Nishida W, Maguchi M, Hiwada K. Autonomic nervous function in non-dipper essential hypertensive subjects. Evaluation by power spectral analysis of heart rate variability. Hypertension 1995; 26: 808-814.

31 Schuler JL, O'Brien WH. Cardiovascular recovery from stress and hypertension risk factors: a meta-analytic review. Psychophysiology 1997; 34: 649-659.

32 Zanzinger J, Czachurski J. Chronic oxidative stress in the RVLM modulates sympathetic control of circulation in pigs. Pflugers Arch 2000; 439: 489-494.

33 Erdos B, Snipes JA, Tulbert CD, Katakam P, Miller AW, Busija DW. Rosuvastatin improves cerebrovascular function in Zucker obese rats by inhibiting $\mathrm{NAD}(\mathrm{P}) \mathrm{H}$ oxidasedependent superoxide production. Am J Physiol Heart Circ Physiol 2006; 290: H1264-H1270.

34 Kvetnansky R, Micutkova L, Rychkova N, Kubovcakova L, Mravec B, Filipenko M, Sabban EL, Krizanova O. Quantitative evaluation of catecholamine enzymes gene expression in adrenal medulla and sympathetic Ganglia of stressed rats. Ann N Y Acad Sci 2004; 1018: 356-369.

35 Dogan MD, Sumners C, Broxson CS, Clark N, Tumer N. Central angiotensin II increases biosynthesis of tyrosine hydroxylase in the rat adrenal medulla. Biochem Biophys Res Commun 2004; 313: 623-626.

36 Tumer N, Larochelle JS. Tyrosine hydroxylase expression in rat adrenal medulla: influence of age and cold. Pharmacol Biochem Behav 1995; 51: 775-780.

37 Takekoshi K, Ishii K, Isobe K, Nanmoku T, Kawakami Y, Nakai T. Angiotensin-II subtype 2 receptor agonist (CGP-42112) inhibits catecholamine biosynthesis in cultured porcine adrenal medullary chromaffin cells. Biochem Biophys Res Commun 2000; 272: 544-550.

38 Jezova M, Armando I, Bregonzio C, Yu ZX, Qian S, Ferrans VJ, Imboden H, Saavedra JM. Angiotensin II AT(1) and AT(2) receptors contribute to maintain basal adrenomedullary norepinephrine synthesis and tyrosine hydroxylase transcription. Endocrinology 2003; 144: 2092-2101.

39 Masuo K, Mikami H, Itoh M, Ogihara T, Tuck ML. Sympathetic activity and body mass index contribute to blood pressure levels. Hypertens Res 2000; 23: 303-310. 